\title{
Comparative efficacy and safety of intravenous and oral administration of a TRH analogue (RX77368) in motor neuron disease
}

\author{
H Modarres-Sadeghi, R J Guiloff
}

\begin{abstract}
Ten consecutive patients with motor neuron disease (MND) who had bulbar symptoms received one or two intravenous doses followed by increasing oral doses of a TRH analogue (RX77368). Similar improvements in speech, swallowing and in tongue and jaw movements were seen after iv and oral administration in nine, five and eight patients respectively. The initial time course of improvement correlated with increasing plasma levels of the drug, but most clinical effects persisted when the levels decreased and became undetectable after 24 hours. The oral solution was tasteless and had no, or minimal, side effects.
\end{abstract}

Department of

Neurology, Charing

Cross and

Westminster Medical

School, Westminster

Hospital, London

H Modarres-Sadeghi

R J Guiloff

Correspondence to:

Dr Guiloff, Department of Neurology, Charing Cross and Westminster Medical School, Westminster

Hospital, 17 Page Street,

London SW1 $2 \mathrm{AF}$, United Kingdom.

Received 14 June 1989 and in revised form 9 February in revised
1990.

Accepted 23 February 1990 ders for a median of 18 days (range 14-90). This study was designed to evaluate the comparative efficacy and safety of oral and iv RX77368 and to establish an oral dose range that would produce a similar profile of effects to an iv dose in MND patients with bulbar syndrome.

\section{Materials and methods}

Ten consecutive patients with MND with bulbar symptoms admitted to hospital were studied (table). The diagnostic criteria have been described previously. ${ }^{34}$ Informed consent was obtained. The study was approved by the local ethical committee. RX77368 (pyroGlu-His-3,3-dimethylProNH2) in both iv and freeze dried form for oral administration was provided by Reckitt and Colman. Oral solutions were prepared by reconstitution of freeze dried RX77368 in tap water to produce a concentration of $1-10 \mathrm{mg} / \mathrm{ml}$. All patients received first one or two doses of iv RX77368 $(0.15-0.25 \mathrm{mg} / \mathrm{kg})$ over a period of two hours. After a subjective and objective response to iv doses was seen, and the majority of the objective measurements were back to the baseline level, they received increasing oral doses of RX77368 $(0.7,1.0$, $1.4,1.8,2.4$ and $3 \mathrm{mg} / \mathrm{kg}$ ). Two patients also received 0.2 and $0.4 \mathrm{mg} / \mathrm{kg}$ orally. The interval between iv and oral doses was three to four days in three patients and six to seven

Table Clinical features of comparative findings in quantitative bulbar function and respiratory measurements for intravenous and oral administration of $R X 77368$

\begin{tabular}{|c|c|c|c|c|c|c|c|c|c|c|c|}
\hline \multirow[b]{2}{*}{ No } & \multirow[b]{2}{*}{ Age } & \multicolumn{6}{|c|}{ Clinical features } & \multicolumn{2}{|l|}{ Improvement } & \multicolumn{2}{|c|}{ Deterioration } \\
\hline & & Form & Dur & $S_{p}$ & $S W$ & $T G$ & $V C$ & $i v$ & oral & $i v$ & oral \\
\hline 1 & $47 M$ & ALS & 38 & 1 & 2 & $\mathrm{~S}_{2} \mathrm{~L}_{1}$ & $2 \cdot 5$ & $\underset{\text { PEP }}{\text { SW, TP» TL*, JM, TIC, }}$ & 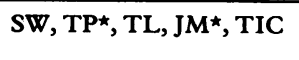 & Nil & MIP, (MEP) \\
\hline 2 & $69 \mathrm{~F}$ & B & 17 & 3 & 3 & $\mathrm{~S}_{3} \mathrm{~L}_{1}$ & $1 \cdot 3$ & $\begin{array}{l}\text { SW, TP, TL*, JM, TIC, } \\
\text { PEP, VC (early) }\end{array}$ & $\underset{\text { PEP }}{S W, T P^{\star}, T L, ~ J M, ~ T I C, ~}$ & $\begin{array}{l}\text { VC (late) } \\
\text { MEP }^{\star}\end{array}$ & VC, MEP^, $\left(T^{\star}{ }^{\star}\right)$ \\
\hline $\begin{array}{l}3 \\
4\end{array}$ & $\begin{array}{l}45 M \\
63 M\end{array}$ & $\begin{array}{l}\mathbf{B} \\
\mathbf{B}\end{array}$ & $\begin{array}{l}14 \\
13\end{array}$ & $\begin{array}{l}3 \\
2\end{array}$ & $\begin{array}{l}1 \\
2\end{array}$ & $\begin{array}{l}S_{3} L_{1} \\
S_{2} L_{1}\end{array}$ & $\begin{array}{l}2 \cdot 4 \\
2 \cdot 7\end{array}$ & $\begin{array}{l}\text { SW, JM } \\
\text { SW, TP*, MIP, MEP* }\end{array}$ & $\begin{array}{l}\mathrm{JM}^{\star} \\
\text { (VC) } \\
\mathrm{TPP}^{\star}, \mathrm{MIP}, \mathrm{MEP}^{\star}\end{array}$ & $\begin{array}{l}\text { TP, PF* } \\
\text { VC, TIC }\end{array}$ & $\begin{array}{l}\mathrm{PF}^{\star}, \mathrm{VC},(\mathrm{JM}, \mathrm{TP}) \\
\mathrm{PF}^{\prime}\left(\mathrm{TP}^{\star}, \mathrm{TIC}^{\star}\right)\end{array}$ \\
\hline $\begin{array}{l}5 \\
6\end{array}$ & $\begin{array}{l}32 \mathrm{M} \\
53 \mathrm{M}\end{array}$ & $\begin{array}{l}\text { ALS } \\
\text { ALS }\end{array}$ & $\begin{array}{l}32 \\
28\end{array}$ & $\begin{array}{l}1 \\
1\end{array}$ & $\begin{array}{l}2 \\
1\end{array}$ & $\begin{array}{l}S_{2} L_{1} \\
S_{1}\end{array}$ & $\begin{array}{l}3 \cdot 3 \\
4 \cdot 3\end{array}$ & 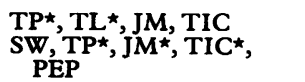 & 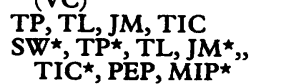 & $\begin{array}{l}\text { VC } \\
\text { PF }\end{array}$ & $\begin{array}{l}\text { VC, PEP, MEP } \\
\text { PF }\end{array}$ \\
\hline $\begin{array}{r}7 \\
8 \\
9 \\
10\end{array}$ & $\begin{array}{l}56 M \\
48 M \\
32 F \\
64 M\end{array}$ & $\begin{array}{l}\text { B } \\
\text { ALS } \\
\text { ALS } \\
\text { ALS }\end{array}$ & $\begin{array}{l}18 \\
96 \\
72 \\
21\end{array}$ & $\begin{array}{l}2 \\
1 \\
2 \\
1\end{array}$ & $\begin{array}{l}1 \\
1 \\
2 \\
1\end{array}$ & $\begin{array}{l}S_{2} L_{1} \\
S_{1} L_{2} \\
S_{1} \\
S_{1}\end{array}$ & $\begin{array}{l}1 \cdot 4 \\
3 \cdot 3 \\
0 \cdot 8 \\
2 \cdot 4\end{array}$ & 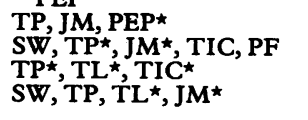 & 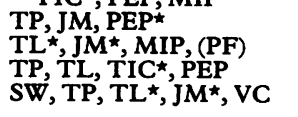 & $\begin{array}{l}\text { TL } \\
\text { Nil } \\
\text { Nil } \\
\text { PF }\end{array}$ & $\begin{array}{l}\text { TL, TIC, (SW) } \\
\text { Nil,(TP) } \\
\text { Nil (PF, TIC) } \\
\text { Nil, (PF, }\end{array}$ \\
\hline
\end{tabular}

$M=$ male, $F=$ female. $B=$ bulbar. Dur = duration of illness in months. $S P=$ speech, $S W=$ swallowing, $T G=$ tongue movements, $S=$ spasticity, $L=$ lower moto neuron involvement.

Impairment grading: $0=$ nil, $1=$ mild, $2=$ moderate, $3=$ severe

$T P$ and $T L=$ timed tongue protrusion and lateral movement.

JM = timed jaw movement. PEP and TIC = timed repetition of the words "pepper" and "ticker".

$\mathrm{VC}=$ mean baseline forced vital capacity (l).

$\mathrm{PF}=$ peak flow. MIP and MEP = maximal inspiratory and expiratory pressure.

* = improvement or deterioration between 4.1-10 SD from mean baseline; no asterisk $=2 \cdot 1-4$ SD from mean baseline.

The parameters in brackets only changed with higher $(1.8-3 \mathrm{mg} / \mathrm{kg})$ oral doses. 
days in seven. The oral doses were given twice per week (interval: $48-120$ hours).

The subjective response to the drug was recordeci. Neurological assessments and measurements of forced vital capacity (VC), peak flow, maximal expiratory and inspiratory pressure (MEP, MIP) as well as timed tongue and jaw movements and word repetition are described elsewhere. ${ }^{34}$ Swallowing $100 \mathrm{ml}$ of water with a straw was also timed. The tests were standardised for each individual. Twenty baseline assessments were made over six to 10 days for all quantitative measure-
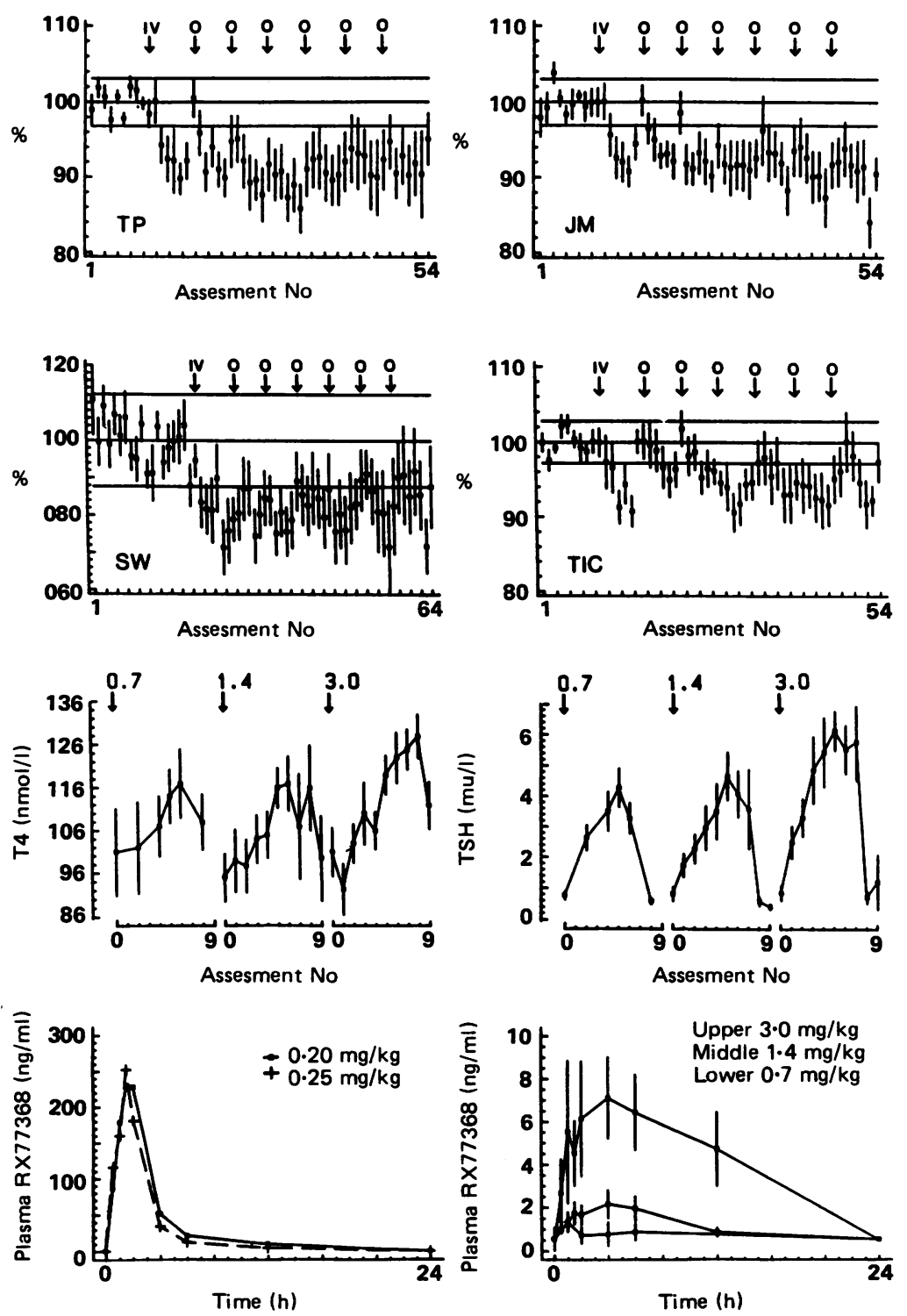

Figure Top two rows: changes in timed tongue protrusion (TP), jaw movement (JM), swallowing (SW) and word repetition (TIC) in all patients receiving iv and oral RX77368. Y Axis: \% change from mean baseline (100\%). Horizontal lines represent two SD from mean baseline. At each assessment point mean $\%$ change in all patients

( $S E$, vertical bars) is shown. IV = intravenous; $O=$ oral. The oral doses were $0 \cdot 7,1 \cdot 0$, $1.4,1.8,2.4$ and $3.0 \mathrm{mg} / \mathrm{kg}$ respectively. $X$ axis: Serial assessment number. The values before the iv dose are baseline values. For all doses, assessments are shown at times 0 (arrows) and after 1, 2, 4, 6 and 24 hours; those 48 hours after iv and 48 and 72 hours after the last oral dose are also plotted. Note significant improvement (that is decrease in time in TP, JM, SW, and TIC) which starts soon after the iv doses and persists or is reproduced with oral doses. Third Row: changes in T4 and TSH following three different oral doses of $R X 77368 ; Y$-axis: Mean (SE) T4 and TSH serum levels. $X$-axis: Numbers 0 to 9 represent samples taken at times $0,0 \cdot 5,1,1 \cdot 5,2,4,6,24$ and 48 hours after each oral dose. The interval between the doses was about one week.

Normal serum levels are: $T 4=(70-160 \mathrm{nmol} / \mathrm{l}), T S H=(0.5-5 \mathrm{mu} / \mathrm{l})$. Bottom row: Plasma concentration of $R X 77368$; left: In two patients receiving a two hour infusion

(IP), right: In seven patients after three single oral doses. The values are mean (SE).

The doses are shown in the top right of each graph. The detection limit of the assay is

$0.56 \mathrm{ng} / \mathrm{ml}$. ments. The best of three replications obtained at each assessment was taken for analysis (for swallowing one replication was obtained). Changes in speech, in range of jaw, tongue, palate and facial movements, in spasticity and in cramps were scored (0-3). ${ }^{4}$ All assessments were performed by the same observer.

Qualitative and quantitative measurements were repeated at times $0,1,2,4,6$ and 24 hours after each dose (iv and oral) and daily afterwards. All quantitative baseline measurements were plotted against time. For parameters which showed learning effects accepting the last 10 baseline assessments made over three to four days was empirically satisfactory. ${ }^{5}$ Values outside two SD of mean baseline were considered as significant change. ${ }^{34}$ Improvement or deterioration was defined as more than two values with significant change in the first three days after each dose. Detection of changes in this way agreed with an analysis by a cumulative sum test and by a regression method. ${ }^{4}$

The quantitative measurements in each patient were expressed as percentage change from individual mean baseline $(100 \%)$ for each parameter because of the wide variation in the baseline values. In this way all clinical courses could be superimposed (fig). Side effects were classified as nil, mild (just noticeable-does not interfere with any activities), moderate (relatively uncomfortable-may interfere with some activities) and severe (uncomfortable-may need treatment). Oral temperature, pulse rate (PR), blood pressure (BP) and respiratory rate (RR) were also monitored. General medical examination and haematology and biochemistry tests were checked 24 hours after each dose. In seven patients serum thyroxine (T4), tri-iodothyronine (T3), thyroid stimulating hormone (TSH), growth hormone (GH), prolactin (PRL) and plasma RX77368 were measured at times $0,1,2,4,6$ and 24 hours after a $0.7 \mathrm{mg} /$ $\mathrm{kg}$ dose and $0.5,1,1.5,2,4,6,12,24$ and 48 hours after 1.4 and $3.0 \mathrm{mg} / \mathrm{kg}$ oral doses. Plasma RX77368 was measured at $0,0.5,1$, $1 \cdot 5,2,4,6,12,24$ and 48 hours after iv doses in two patients. The samples for hormone and drug levels were centrifuged and the plasma separated and stored at $-20 \mathrm{C}$ within 30 minutes.

\section{$R X 77368$ immunoassay}

This assay was developed by Mr A Lynn (Reckitt and Colman) and Dr G Bennett (Nottingham University). Because RX77368 is a nonimmunogenic tripeptide it was linked with a large molecular weight protein (keyhole limpet haemocyanin). The conjugate was injected into two sheep at monthly intervals. Iodinated RX77368 radiolabel was used for analysis of test bleeds. Cross reactivity studies with several TRH analogues and potential metabolites of RX77368 showed that the antibodies were highly specific for RX77368. The only compound that exhibited any appreciable crossreactivity was RX74355 which has only one methyl substitution in the prolineamide fragment of TRH. Animal work 
indicates that $\mathrm{RX} 74355$ is not a breakdown product of RX77368. The interassay mean coefficient of variation was $10 \%$ (intra-assay $1.5 \%$ ).

\section{Results}

Bulbar functions

The time course of the mean $\%$ change in timed tongue and jaw movements, swallowing and word repetition is given in the figure. The table summarises the changes in individual patients after iv and oral administration.

All patients showed improvement in speech after iv doses, which was graded as three in seven cases, as two in two and as one in another. The patients described clearer and less laborious speech, lighter tongue and a stronger voice. The median (and range) onset and duration of improvement was two (1-24) and 48 (24-96) hours with iv doses. The improvement was reproduced in nine patients after oral doses. The median onset of improvement after the oral doses was four (224) hours and it persisted throughout the study period and up to two weeks after the last dose in four cases. In the other five the median duration of improvement after each dose was 24 hours (8-48); with higher oral doses (1.8-3 $\mathrm{mg} / \mathrm{kg}$ ) they showed an initial deterioration (median onset 2, range $1-6$ and duration 6 , range $2-8$ hours) followed by improvement 24 hours later in four of them.

Seven patients showed subjective and objective improvement in swallowing after iv doses. Five of them showed similar improvement after oral doses of $0.7 \mathrm{mg}$ or more (table). Solid food was specially easier to swallow. One patient was able to eat food she had been unable to eat for the previous six months. The others described less tendency to choke, easier to clear throat and more confidence when eating. The median (and range) onset and duration of subjective and objective improvement with iv doses were four (1-48) and 48 (12-96) hours respectively. With oral doses the median onset of improvement was six (1-24) hours. This was maintained throughout the study period and for up to 10 and 14 days after the last oral dose in two patients. In the others the maximum duration of improvement after each dose ranged from three to four days and, in one case, deterioration lasted for 24 hours after higher oral doses.

Slight improvement in the range of jaw or tongue movements was seen in two patients, only after iv doses. No change in the range of facial and palatal movements was seen.

\section{Respiration}

There were no subjective changes in breathing. The effects on objective measurements were not consistent. Individually the mean percentage change (values outside two standard deviations of mean baseline) ranged from $16 \%$ to $44 \%$ (maximum duration of change are to seven days) for deterioration and $17 \%$ to $67 \%$ (one to 10 days) for improvement. Deterioration in both PF and VC was seen in two of the patients.

\section{Cramps and spasticity}

Cramps improved in three patients (one from grade 3 to 1 , others grade 2 to 1 ). This started after the iv dose and was maintained throughout the study period and for up to four weeks after the last oral dose. Others did not have them or had occasional ones which remained unchanged. All three patients who had spasticity in the limbs showed mild subjective and objective improvement; it was most evident 24 hours after iv or oral doses with a mean duration of 24 hours, although in one case improvement persisted throughout the study period and up to one week after the last dose. These changes were not associated with major objective functional changes but two of them reported improvement in their walking and in the other the range of arm movements improved.

\section{Other changes}

No change was noted in MRC grading, deep tendon and plantar reflexes nor in the Norris scale although there was subjective improvement in neck strength in one patient and in arm strength in another at the end of treatment period.

\section{Side effects}

The oral solution was tasteless and well tolerated. Side effects were graded as nil to mild in all patients with different oral doses and as moderate to severe in four patients with iv doses. These have been described previously. ${ }^{34}$ The median (and range) onset and duration of side effects after iv and oral doses were one $(0 \cdot 1-4)$ and six (2-10) hours and $1 \cdot 5(0 \cdot 2-10)$ and four $0 \cdot 1-10)$ hours respectively. No relation was found between the side effects and the clinical response or the endocrine changes. BP, $\mathrm{PR}, \mathrm{RR}$, oral temperature, and haematology and biochemistry tests remained normal.

\section{Endocrine results}

There was a temporary increase in $\mathrm{TSH}, \mathrm{T} 4$ and $\mathrm{T} 3$ following three different oral doses (fig). Serum prolactin peaked at 0.5 hour. The levels returned to the baseline range within 24 hours. Changes in $\mathrm{GH}$ were less consistent but there was an overall increase with a peak level at 1.5 hours. All values remained within the normal range except for a transient increase in $\mathrm{TSH}$ in all and $\mathrm{GH}$ in one patient.

\section{$R \times 77368$ plasma levels}

The peak plasma drug levels after oral doses ranged from 3.8 to $27 \mathrm{ng} / \mathrm{ml}$. After stopping the infusions the plasma levels declined to around $10 \mathrm{ng} / \mathrm{ml}$ over the next 10 hours, and at 24 hours were below the level of detection. After the oral doses the peak levels were achieved at four hours and were much less (below $10 \mathrm{ng} / \mathrm{ml}$ in most patients) than with iv doses; they declined gradually and were undetectable at 24 hours (fig). The onset of the effects on bulbar function correlated with increasing plasma levels but the degree and duration of effects had no relationship to them. The time to peak of plasma RX77368 with oral doses was the same 
as that of serum TSH but longer that of prolactin and shorter than those of T3 and T4.

\section{Discussion}

The improvements in speech, swallowing, spasticity and cramps seen with iv RX77368 could be reproduced or maintained with oral doses in most patients during the study $\operatorname{period}(3.5$ weeks). The relationship between the initial period of improvement, and of side effects, to the increase in drug plasma levels after each dose and the lack of any response to small $(0.2-0.4 \mathrm{mg} / \mathrm{kg})$ oral doses in two cases make a placebo effect unlikely. Further, the response to the oral drug was similar to that of iv doses seen in this study and previously. ${ }^{34} \mathrm{~A}$ carry-over effect is unlikely since the drug was given orally three to seven days after the iv doses, when most of the bulbar measurements were back to the baseline range.

The changes seen in speech and bulbar function occurred mainly in patients with spastic dysarthria and might be related to decreased spasticity by a central or peripheral action or to effects on lower brain stem nuclei and/or their connections.

The plasma levels of the drug were undetectable 24 hours after iv administration. The persistence of improvement in bulbar function with undetectable blood levels of the analogue is intriguing. The half life of RX77368 in human brain stem is not known, but in brain it was found to be 168 minutes. ${ }^{1}$ If its metabolism is the same in MND patients, and RX77368 acts centrally, the prolonged effects suggest that its action is mediated by other substances (including its metabolites). Alternatively RX77368 might induce longer duration changes in the relevant neuronal populations.

The detection of the analogue in the plasma within 0.5 hour of ingestion and endocrine changes suggest rapid absorption. The optimum oral dose appeared to be in the middle of the range used with an oral/iv ratio of around seven. Some patients showed an initial deterioration in speech (followed by improvement), or a deterioration in swallowing or in other quantitative measurements when the dose was further increased. The degree of improvement was slight to moderate. This was despite the low plasma levels of the analogue achieved with oral doses and may point to the presence of a ceiling level for therapeutic response. There was no relationship between the degree of improvement and the extent of disability of the patients though the effect on speech was less noticeable in those with severe dysarthria. The lack of severe side effects observed with oral doses is likely to be related to the lower peak serum levels but receptor down regulation may have also contributed. ${ }^{67}$ A study of the timing of side effects in previous patients receiving iv $\mathrm{Rx} 77368$ and plasma drug levels obtained in this study indicates that the majority of moderate to severe side effects occur with plasma levels above around $10 \mathrm{ng} /$ $\mathrm{ml}$.

The finding of a similar profile of effects after iv and oral doses, the lack of, or minimal, side effects of oral administration and the fact that the solution is almost tasteless suggest that it may be suitable for longterm studies. The short term clinical benefit is small or moderate, though not unimportant, and sensitive methods are necessary to detect the changes. If the beneficial effects seen could be maintained for longer periods, oral RX77368 may have a place as symptomatic treatment for MND patients with bulbar syndrome. Since death in most MND patients is related to bulbar and high cervical cord involvement the possibility of modifying survival time should be carefully considered even if somatic disability remains unchanged. ${ }^{45}$

We thank Drs JP Ballantyne, LP Blumhardt, P Bradbury, RAC Hughes, N Hyman, J Jestico, C Kennard and R McKeran who kindly referred patients for this study. This work was supported by grants from the NW Thames Regional Health Authority and the Special Trustees of Westminster and Roehampton Hospitals. We are also grateful to the Chemical Pathology Department for the endocrine assays, to Mr A Lynn (Reckitt and Colman Pharmaceutical Division, Hull, United Kingdom) for
measuring plasma RX77368 levels and to the Pharmacy for preparing the oral solutions.

1 Guiloff RJ. Thyrotropin releasing hormone and motorneurone disease. Reviews in the Neurosciences 1987; 1(3-4):201-19.

2 Guiloff RJ. Use of TRH analogues in Motorneuron Disease. In: Thyrotropin-Releasing Hormone. Biomedical significance. Edited by $\mathrm{G}$ Metcalf and IVD Jackson. $A n n N$ Y Acad Sci 1989;553:399-421.

3 Guiloff RJ, Eckland DJA, Demain C, et al. Controlled Acute Trial of a Thyrotropin-Releasing Hormone Analogue Trial of a Thyrotropin-Releasing Hormone Analogue
(RX77368) in Motor Neurone Disease. J Neurol (RX77368) in Motor Neurone Dis
Neurosurg Psychiatry 1987;50:1359-70.

4 Modarres-Sadeghi HR, Rogers H, Emami J, Guiloff RJ. Subacute administration of a TRH analogue (RX77368) in motorneuron disease: an open study. $J$ Neurol Neurosurg Psychiatry 1988;51:1146-57.

5 Guiloff RJ, Modarres-Sadeghi HR, Rogers H. Motorneurone Disease: Aims and assessment methods in trial design. In: Clifford-Rose F, ed. Clinical trials in Amyotrophic lateral sclerosis. Demos: New York 1989:13-21.

6 Ogawa N, Mizumo S, Nukina I, et al. Chronic thyrotropin releasing hormone (TRH) administration on TRH receptors and muscarinic cholinergic receptors in CNS. Brain Res 1983;263:348-50.

7 Simasko SM, Horita A. Treatment of rats with a TRH analogue MK-771. Down Regulation of TRH receptors and Behavioural Tolerance. Neuropharmacology 1985;24:157-65. 